\title{
General Shape Control of Colloidal CdS, CdSe, CdTe Quantum Rods and Quantum Rod Heterostructures
}

\author{
Felice Shieh $^{\dagger}$, Aaron E. Saunders ${ }^{\dagger}$, Brian A. Korgel* \\ Department of Chemical Engineering, Texas Materials Institute, Center for Nano- and Molecular \\ Science and Technology, University of Texas, Austin, Texas 78712 \\ ${ }^{\dagger}$ These authors contributed equally to this work. \\ * Corresponding author: (T) 512-471-5633; (F) 512-471-7060; korgel@mail.che.utexas.edu
}

\section{Nanocrystal Synthesis}

The cadmium precursor is prepared by degassing a mixture of $0.114 \mathrm{~g} \mathrm{CdO}(0.89 \mathrm{mmol})$, $0.43 \mathrm{~g}$ n-tetradecylphosphonic acid (TDPA) $(0.155 \mathrm{mmol})$ and $7 \mathrm{~g}$ of trioctylphosphine oxide (TOPO) at $65{ }^{\circ} \mathrm{C}$ for three hours. The mixture is heated to $340{ }^{\circ} \mathrm{C}$ under nitrogen. At temperatures above $300{ }^{\circ} \mathrm{C}$, the solution gradually turns from a dark red to a clear colorless solution, indicating the formation of the Cd-TDPA complex. The mixture is then cooled to the desired injection temperature: $300{ }^{\circ} \mathrm{C}$ for $\mathrm{CdTe}$ and $\mathrm{CdS}$, and $260{ }^{\circ} \mathrm{C}$ for $\mathrm{CdSe}$. The chalcogen precursor is prepared by dissolving $1 \mathrm{mmol}$ of $\mathrm{S}$, Se or Te powder in $5 \mathrm{~mL}$ of trioctylphosphine (TOP) and heating to $120^{\circ} \mathrm{C}$.

Spherical particles are synthesized by rapid injection of the chalcogen-TOP precursor. Rods are synthesized by injecting the chalcogen precursor in $0.5 \mathrm{~mL}$ volumes periodically, every 3 minutes for CdSe and every 2 minutes for CdTe and CdS. The highest aspect ratio CdSe and CdTe nanorods are typically recovered after four or five injections. CdS nanorod growth is slowest, and the highest apect ratio rods are obtained after the final injection. Heterostructure nanorods are grown by alternating the chalcogen precursor during sequential injection. $\mathrm{CdTe} / \mathrm{CdSe} / \mathrm{CdTe}$ nanorods are prepared by synthesizing CdSe rods from four Se-TOP injections three minutes apart followed by three or four Te-TOP injections two minutes apart. 
$\mathrm{CdS} / \mathrm{CdTe} / \mathrm{CdS}$ nanorods are grown by four sequential Te-TOP injections two minutes apart followed by eight S-TOP injections two minutes apart.

The nanocrystals and nanorods are purified by precipitating the particles with excess ethanol and discarding the supernatant after centrifugation. The nanocrystals can be dispersed in organic solvents, such as hexane and chloroform, for characterization.

\section{Characterization methods}

TEM images were obtained using either a Phillips EM280 microscope operated at an accelerating voltage of $80 \mathrm{kV}$ or a JEOL $2010 \mathrm{~F}$ electron microscope equipped with a field emission gun operated at $200 \mathrm{kV}$. The TEM samples were prepared by drop casting purified nanocrystals from hexane on a carbon-coated copper TEM grid (Ladd Research). Powder X-ray diffraction patterns were collected using a Bruker-Nonius D8 Advance powder diffractometer from samples prepared by drying a thick nanocrystal film on a quartz substrate. Absorbance spectra were collected using an Aligent 8453 spectrophotometer and photoluminescence spectra were recorded using a Fluorlog-3 fluorimeter with a 450W Xe source. 